\title{
Networking by small-molecule hormones in plant immunity
}

\author{
Corné M J Pieterse, Antonio Leon-Reyes, Sjoerd Van der Ent \& Saskia C M Van Wees
}

\begin{abstract}
Plants live in complex environments in which they intimately interact with a broad range of microbial pathogens with different lifestyles and infection strategies. The evolutionary arms race between plants and their attackers provided plants with a highly sophisticated defense system that, like the animal innate immune system, recognizes pathogen molecules and responds by activating specific defenses that are directed against the invader. Recent advances in plant immunity research have provided exciting new insights into the underlying defense signaling network. Diverse small-molecule hormones play pivotal roles in the regulation of this network. Their signaling pathways cross-communicate in an antagonistic or synergistic manner, providing the plant with a powerful capacity to finely regulate its immune response. Pathogens, on the other hand, can manipulate the plant's defense signaling network for their own benefit by affecting phytohormone homeostasis to antagonize the host immune response.
\end{abstract}

Phytohormones are small molecules that are essential for the regulation of plant growth, development, reproduction and survival. They act as signal molecules and occur in low concentrations. Classic phytohormones are abscisic acid (ABA), auxins, cytokinins, ethylene (ET) and gibberellins, but small signaling molecules such as brassinosteroids, jasmonates (JAs) and salicylic acid (SA) are recognized as phytohormones as well ${ }^{1}$. Changes in hormone concentration or sensitivity, which can be triggered under biotic and abiotic stress conditions, mediate a whole range of adaptive plant responses. The importance of SA, JAs and ET as primary signals in the regulation of the plant's immune response is well established ${ }^{2-6}$. More recently, $\mathrm{ABA}^{7,8}$, auxins $s^{9,10}$, gibberellins ${ }^{11}$, cytokinins $s^{12,13}$ and brassinosteroids ${ }^{14,15}$ emerged as players on the battle field as well (Fig. 1). The involvement of so many plant growth regulators in plant immunity suggests that the control of plant growth, development and defense is interconnected in a complex network of cross-communicating hormone signaling pathways. The great regulatory potential of such a network may allow plants to quickly adapt to their biotic and abiotic environment and to utilize their resources in a cost-efficient manner. It is generally believed that hormone-regulated induced defense responses evolved to save energy under enemy-free conditions, as they only involve costs when defenses are activated upon pathogen or insect attack ${ }^{16}$. These costs arise from the allocation of resources to defense and away from plant growth and development. Trade-offs between plant growth rate and disease resistance have been well documented ${ }^{16}$ and support the hypothesis that plant growth and defense are regulated by a network of interconnecting signaling pathways.

Upon pathogen attack, the quantity, composition and timing of the phytohormonal blend produced by the plant varies among plant species

Plant-Microbe Interactions, Department of Biology, Faculty of Science, Utrecht University, Utrecht, The Netherlands. Correspondence should be addressed to C.M.J.P. (c.m.j.pieterse@uu.nl).

Published online 17 April 2009; doi:10.1038/nchembio.164 and depends greatly on the lifestyle and infection strategy of the invading attacker. This 'signal signature' results in the activation of a specific set of defense-related genes that eventually determines the nature and effectiveness of the immune response that is triggered by the attacker ${ }^{17}$. In recent years, molecular, genetic and genomic tools have been used to uncover the complexity of the hormone-regulated induced defense signaling network. Besides balancing the relative abundance of different hormones, intensive interplay between hormone signaling pathways emerged as an important regulatory mechanism by which the plant may be able to tailor its immune response to the type of invader encountered. On the other hand, evidence is accumulating that pathogens can manipulate hormone-regulated signaling pathways to evade host immune responses. Here we review our current understanding of the roles of phytohormones in the plant's immune system, with a focus on crosstalk between defense hormone signaling pathways and its significance in plant-pathogen interactions.

\section{The plant immune system}

In nature, plants are continuously threatened by a wide range of harmful pathogens and pests, including viruses, bacteria, fungi, oomycetes, nematodes and insect herbivores. Each of these attackers exploits highly specialized features to establish a parasitic relationship with its host plant. According to their lifestyles, plant pathogens are generally divided into necrotrophs and biotrophs (Fig. 2) ${ }^{18}$. Necrotrophs first destroy host cells, often through the production of phytotoxins, after which they feed on the contents. Biotrophs derive nutrients from living host tissues, commonly through specialized feeding structures (haustoria) that invaginate the host cell without disrupting it. Many plant pathogens display both lifestyles, depending on the stage of their life cycle, and are called hemibiotrophs.

To defend themselves against all these different types of pathogens, plants have an array of structural barriers and preformed antimicrobial metabolites to prevent or attenuate invasion by potential attackers. Despite the diversity of these constitutive defenses, many microbes 
succeed in breaking through this pre-invasive layer of defense. However, a broad spectrum of inducible plant defenses can be recruited to limit further pathogen ingress. For this postinvasive line of defense, plants have evolved sophisticated strategies to perceive their attacker and to translate this perception into an effective immune response ${ }^{19}$. First, the primary immune response recognizes common features of microbial pathogens, such as flagellin, chitin, glycoproteins and lipopolysaccharides ${ }^{20,21}$. These microbial determinants are referred to as pathogen-associated molecular patterns (PAMPs) ${ }^{19,20,22}$. PAMPs activate pattern-recognition receptors (PRRs), which in turn initiate diverse downstream signaling events that ultimately result in the activation of a basal resistance that is called PAMP-triggered immunity (PTI; Fig. 3a) ${ }^{19,22}$. During the co-evolutionary arms race between pathogens and their host plants, pathogens acquired effector molecules that are transported into the host cell to suppress PTI and promote virulence of the pathogen, resulting in effectortriggered susceptibility (ETS; Fig. 3b). In turn, plants acquired resistance (R) proteins that recognize these attacker-specific effectors, resulting in a secondary immune response called effector-triggered immunity (ETI; Fig. 3c) ${ }^{19,22}$. Ultimately, the final outcome of the battle depends on the balance between the ability of the pathogen to suppress the plant's immune system and the capacity of the plant to recognize the pathogen and to activate effective defenses.

\section{Induced plant defense responses}

Many early signaling components of PTI and ETI have been identified in recent years ${ }^{20,21,23}$. Downstream of these early signaling events, plants respond by activating a large number of integrated defense responses to ward off the invader. The nature of the defense responses that are activated during PTI and ETI show substantial overlap ${ }^{24}$. These defenses include cell wall fortification through the synthesis of callose and lignin; the production of antimicrobial secondary metabolites, such as phytoalexins; and the accumulation of pathogenesis-related (PR) proteins, such as chitinases and glucanases, that degrade fungal and oomycete cell walls. Recognition of pathogen-specific effectors through the ETI system is particularly effective because it is followed by a burst of reactive oxygen species that culminates in a programmed hypersensitive cell death at the site of pathogen invasion, keeping the pathogen isolated from the rest of the plant and preventing further damage ${ }^{25}$. Obviously, this hypersensitive response would favor growth of pathogens with a necrotrophic lifestyle, as their virulence strategy relies on their capacity to kill host cells ${ }^{18}$. Therefore, the hypersensitive response is believed to be typically active against pathogens with a biotrophic lifestyle ${ }^{18}$. Immune responses that are active against necrotrophs are likely to be initiated in response to the action of pathogen-derived toxins or damage-associated molecular patterns, such as breakdown products of the plant cell wall that, upon release by the activity of pathogen-derived cell wall-degrading enzymes, can stimulate plant defense responses ${ }^{21,23}$.

The regulation of the defense network that translates the pathogeninduced early signaling events into activation of effective defense responses depends profoundly on the action of phytohormones. Pathogen infection stimulates the plant to synthesize one or more hormonal signals depending on the type of attacker ${ }^{17}$. Compelling evidence for the key role of phytohormones in the plant's immune response comes from studies with the model plant species Arabidopsis thaliana (Arabidopsis) and Nicotiana tabacum (tobacco), in which various mutants and transgenic lines impaired in hormone biosynthesis, perception or signaling were demonstrated to display a severe alteration in the level of resistance to specific types of pathogens. From these studies, it became evident that biotrophic pathogens are generally sensitive to defense responses that are regulated by SA, whereas pathogens with a necrotrophic lifestyle are commonly deterred by defenses that are controlled by JAs and $\mathrm{ET}^{18,26}$. In analogy to the defense response to necrotrophs, the wound response that is effective against insect herbivores is also regulated by the JA signaling pathway ${ }^{5}$.

\section{Systemic immunity}

Once plant defense responses are activated at the site of infection, a systemic defense response is often triggered in distal plant parts
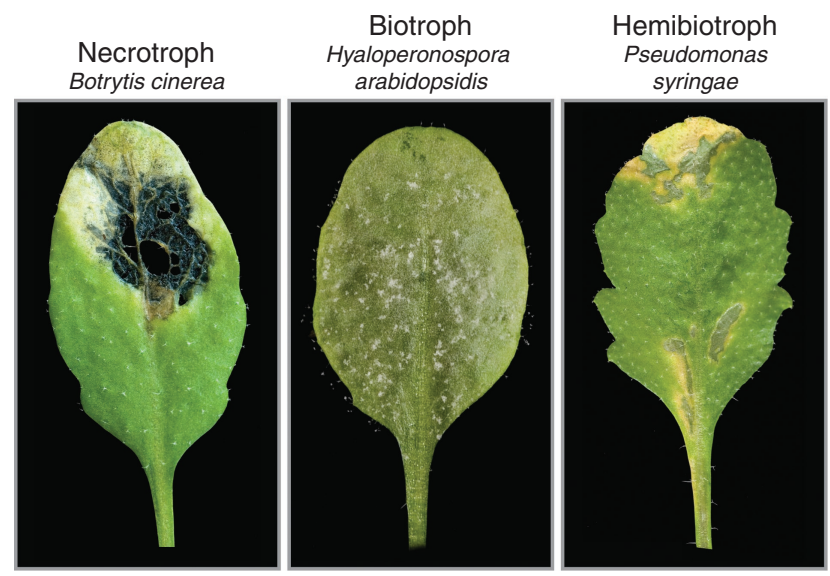

Figure 2 Disease symptoms on Arabidopsis leaves caused by the necrotrophic fungus Botrytis cinerea, the biotrophic oomycete Hyaloperonospora arabidopsidis and the hemibiotrophic bacterium Pseudomonas syringae. Photos: Hans van Pelt. 
a

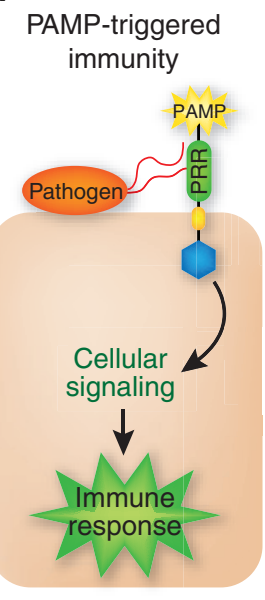

b

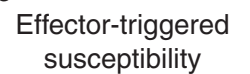

C

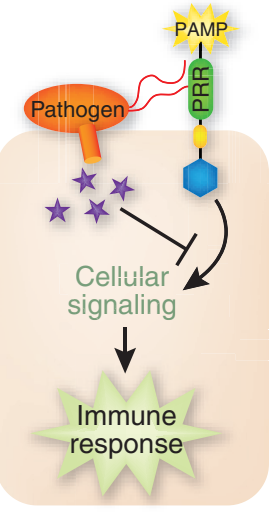
susceptibility

Figure 3 Simplified schematic representation of the plant immune system. (a) Upon pathogen attack, pathogen-associated molecular patterns (PAMPs) activate pattern-recognition receptors (PRRs) in the host, resulting in a downstream signaling cascade that leads to PAMP-triggered immunity $(\mathrm{PTI})^{19}$. (b) Virulent pathogens have acquired effectors (purple stars) that suppress PTI, resulting in effector-triggered susceptibility (ETS). (c) In turn, plants have acquired resistance $(R)$ proteins that recognize these attackerspecific effectors, resulting in a secondary immune response called effectortriggered immunity (ETI).

to protect these undamaged tissues against subsequent invasion by the pathogen. This long-lasting and broad-spectrum induced disease resistance is referred to as systemic acquired resistance (SAR; Fig. 4$)^{27}$ and is characterized by the coordinate activation of a specific set of $P R$ genes, many of which encode for proteins with antimicrobial activity $^{28}$. The onset of SAR can be triggered by PTI- and ETI-mediated pathogen recognition and is associated with increased levels of SA, locally at the site of infection and often also systemically in distant tissues ${ }^{24,29}$. Mutant and transgenic plants that are impaired in SA signaling are incapable of developing SAR and do not show $P R$ gene activation upon pathogen infection ${ }^{27}$, which indicates that SA is a necessary intermediate in the SAR signaling pathway. The regulatory protein NPR1 (NONEXPRESSOR OF PR GENES1) emerged as an important transducer of the SA signal; upon activation by SA, NPR1 acts as a transcriptional co-activator of $P R$ gene expression ${ }^{30}$. The nature of the systemically transported mobile signal that travels from the site of infection to establish SAR in distal tissues is one of the holy grails in plant defense signaling research. Recent studies point to a role for methyl-SA, JAs, a plastid glycerolipid-based factor, and a lipid-transfer protein (reviewed in ref. 31).

Beneficial soil-borne microorganisms, such as mycorrhizal fungi and plant growth-promoting rhizobacteria, can induce a phenotypically similar form of systemic immunity called induced systemic resistance (ISR; Fig. 4) $)^{32,33}$. Like PAMPs of microbial pathogens, different beneficial microbe-associated molecular patterns are recognized by the plant, which results in a mild but effective activation of the immune response in systemic tissues ${ }^{34-36}$. In contrast to SA-dependent SAR, ISR triggered by beneficial microorganisms is often regulated by JA- and ET-dependent signaling pathways and is associated with priming for enhanced defense rather than direct activation of defense $\mathrm{s}^{34,37,38}$. Whereas SAR is predominantly effective against biotrophic pathogens that are sensitive to SA-dependent defenses, ISR was shown to be effective against pathogens and insects that are sensitive to JA- and ET-dependent defenses ${ }^{39,40}$.

\section{Pathway crosstalk to fine-tune defense}

In nature, plants often deal with simultaneous or subsequent invasion by multiple aggressors and beneficials, which can influence the primary induced defense response of the host plant ${ }^{41-43}$. Activation of plant defense mechanisms is associated with ecological fitness costs ${ }^{16}$. Hence, plants need regulatory mechanisms to effectively and efficiently adapt to changes in their complex environment. Crosstalk between hormonal signaling pathways provides the plant with such a powerful regulatory potential and may allow the plant to tailor its defense response to the invaders encountered ${ }^{44-47}$.

The importance of SA, JAs and ET as dominant primary signals in local and systemic induced defense signaling has been well document$\mathrm{ed}^{2-4}$. In recent years, research on their biosynthesis pathways and the way they are perceived by other biomolecules significantly advanced our understanding of the signaling pathways that these small molecules regulate (a simplified schematic representation of the SA, JA and ET signaling pathways is provided in Fig. 5 $)^{4,48,49}$. However, the way these signal molecules function in a complex network of interacting pathways is less well studied. Early work in tomato (Solanum lycopersicum) and Arabidopsis demonstrated that SA and its acetylated derivative aspirin are strong antagonists of the JA signaling pathway ${ }^{50}$, and that JA and ET signaling can act synergistically ${ }^{51}$. The genomics era provided a wealth of new opportunities to investigate how the SA, JA and ET signaling pathways are interconnected in the induced defense signaling network ${ }^{52}$. Whole-genome expression profiling of a large set of Arabidopsis mutants affected in SA, JA or ET signaling in response to infection by the hemibiotrophic bacterial pathogen Pseudomonas syringae ${ }^{53}$ confirmed that there is extensive crosstalk between the SA, JA and ET response pathways and paved the way to model the network topology of the plant's immune response $\mathrm{s}^{52}$.

One of the best studied examples of defense-related signal crosstalk is the antagonistic interaction between the SA and JA response pathways. Many cases of trade-offs between SA-dependent resistance against biotrophic pathogens and JA-dependent defense against necrotrophic pathogens and insect herbivory have been documented $^{41,47}$. For example, induction of the SA pathway in Arabidopsis by the biotrophic oomycete pathogen Hyaloperonospora arabidopsidis strongly suppressed JA-mediated defenses that were activated upon feeding by caterpillars of the small cabbage white Pieris rapae ${ }^{54}$. Activation of the SA pathway by $P$. syringae similarly suppressed JA signaling and rendered infected leaves more susceptible to the necrotrophic fungus Alternaria brassicicola ${ }^{55}$.

Pharmacological experiments with Arabidopsis revealed that JA-responsive marker genes, such as PDF1.2 and VSP2, are highly sensitive to suppression by exogenous application of $\mathrm{SA}^{54,56}$. SA-mediated suppression of JA-responsive gene expression was observed in a large number of Arabidopsis accessions collected from very different geographic origins, which highlights the potential significance of this phenomenon in the regulation of induced plant defenses in nature ${ }^{54}$. Although many reports describe an antagonistic interaction between SA- and JA-dependent signaling, synergistic interactions have been described as well ${ }^{57-59}$. For example in Arabidopsis, treatment with low concentrations of JA and SA resulted in a synergistic effect on the JA- and SA-responsive genes PDF1.2 and $P R-1$, respectively. However, at higher concentrations the effects were antagonistic, demonstrating that the outcome of the SA-JA interaction is dependent on the relative concentration of each hormone $\mathrm{e}^{58}$. Koornneef et al. ${ }^{54}$ demonstrated that timing and sequence of initiation of SA and JA signaling are also important for the outcome of the SA-JA signal interaction. Hence, the kinetics of phytohormone biosynthesis and signaling during the interaction of a plant with its 
attackers could be highly decisive in the final outcome of the defense response to the attacker encountered. Additionally, phytohormones can be readily modified into derivatives with altered biological activity, which adds yet another layer of regulation.

\section{Signaling nodes in the SA-JA-ET network}

So far, many examples of positive and negative crosstalk between SA, JA and ET signaling have been reported and are documented in a series of informative reviews ${ }^{60-65}$. A number of key signaling nodes emerged from these studies and will be highlighted in the following sections according to their role in SA-JA, JA-ET or ET-SA crosstalk (Fig. 6).

SA-JA. In recent years, several proteins with an important regulatory role in SA-JA crosstalk have been identified in Arabidopsis. Mutation or ectopic expression of the corresponding genes were shown to have contrasting effects on SA and JA signaling and on resistance against biotrophs and necrotrophs (reviewed in ref. 60). Among these molecular players are the mitogen-activated protein kinase MPK4 (ref. 66), the lipase-like proteins EDS1 (ENHANCED DISEASE SUSCEPTIBILITY1) and PAD4 (PHYTOALEXIN-DEFICIENT4) ${ }^{67}$, the defense regulatory protein NPR1 (ref. 56), the fatty acid desaturase SSI2 (SUPPRESSOR OF SA INSENSITIVITY2) ${ }^{68}$, the glutaredoxin GRX480 (ref. 69) and WRKY transcription factor proteins such as WRKY70 (ref. 70) (Fig. 6). The majority of the identified crosstalk regulators play pivotal roles in SA signal transduction, in which NPR1 plays a central role. NPR1 acts downstream of EDS1 and PAD4 in the SA signaling pathway ${ }^{67}$. In addition, NPR1 regulates the SA-mediated expression of GRX480 and WRKY70, which encode proteins that suppress JA-dependent gene expression ${ }^{69,70}$. By contrast, SSI2 was identified in a screen for suppressors of the mutant npr1-5 phenotype ${ }^{68}$ and thus exerts an NPR1-independent role in the regulation of SA-JA crosstalk. Mutant ssi2 plants are defective in stearoyl-ACP desaturase, which results in an altered fatty acid content. Mutations that restored the lowered 18:1 fatty acid levels rescued the ssi2 mutant phenotype, which suggests a role for fatty acid signaling in SA-JA crosstalk ${ }^{68,71}$.

Changes in the cellular redox state play a major role in the transduction of the SA signal ${ }^{30,72}$. In Arabidopsis, the ability of SA to suppress JA-responsive genes was shown to coincide with an increase in the level of glutathione, a major determinant of cellular redox homeostasis $^{54}$. The glutathione biosynthesis inhibitor L-buthionine sulfoximine strongly reduced the suppression of the JA-responsive gene PDF1.2 by SA, which suggests that SA-mediated modulation of the cellular redox state is an important trigger for the attenuation of JA signaling $^{54}$. The NPR1 protein is an important transducer of SA-induced redox changes. Besides functioning as a crucial transcriptional coactivator of SA-responsive $P R$ genes ${ }^{30}$, NPR1 is also a key regulator in SA-mediated suppression of JA signaling ${ }^{56,73}$. So how does NPR1 exert its dual role in the activation of SA-responsive genes on the one hand and the suppression of JA-responsive genes on the other hand? SA-induced redox changes activate NPR1 by reducing inactive NPR1 oligomers to active monomers ${ }^{30}$. Active NPR1 monomers are translocated to the nucleus, where they interact with TGA transcription factors that activate SA-responsive genes ${ }^{30,72}$. Interestingly, nuclear localization of SA-activated NPR1 is not required for the suppression of JA-responsive genes, which indicates that the antagonistic effect of SA on JA signaling is modulated through a function of NPR1 in the cytosol $^{56}$. In rice (Oryza sativa), a similar cytosolic function of NPR1 in SA-JA crosstalk was reported ${ }^{74}$. Glazebrook et al. provided additional evidence for a differential role of cytosolic and nuclear NPR1 in the regulation of JA/ET- and SA-dependent signaling, respectively.

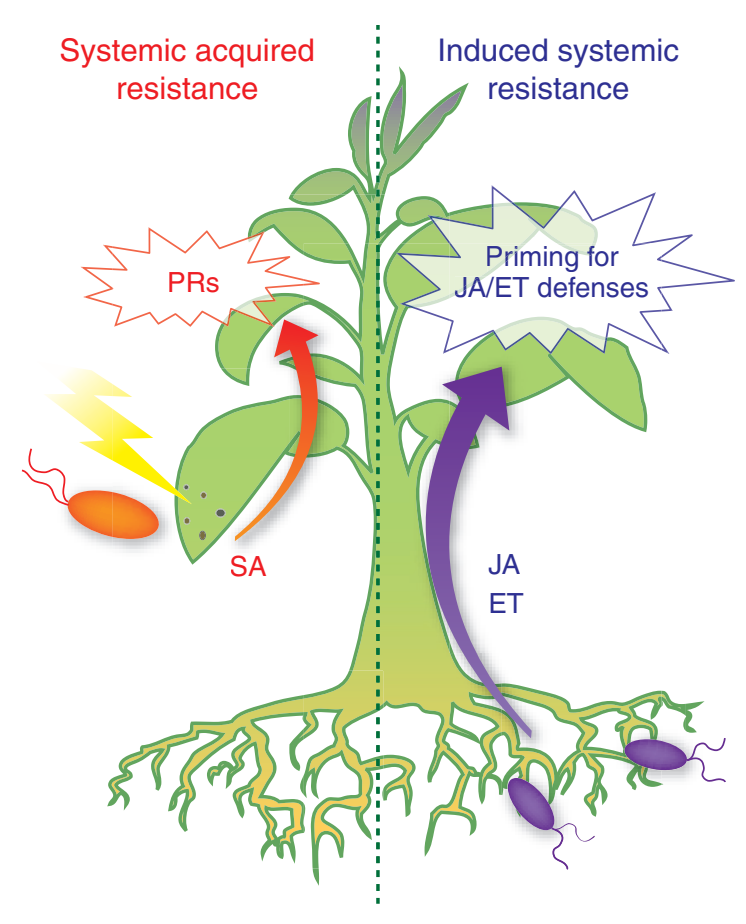

Figure 4 Schematic representation of systemically induced immune responses. Systemic acquired resistance (SAR) is typically activated in healthy systemic tissues of locally infected plants. Upon pathogen infection, a mobile signal travels through the vascular system to activate defense responses in distal tissues. Salicylic acid (SA) is an essential signal molecule for the onset of SAR, as it is required for the activation of a large set of genes that encode pathogenesis-related proteins (PRs) with antimicrobial properties. Induced systemic resistance (ISR) is typically activated upon colonization of plant roots by beneficial microorganisms. Like SAR, a longdistance signal travels through the vascular system to activate systemic immunity in above-ground plant parts. ISR is commonly regulated by jasmonic acid (JA)- and ethylene (ET)-dependent signaling pathways and is typically not associated with the direct activation of $P R$ genes. Instead, ISR-expressing plants are primed for accelerated JA- and ET-dependent gene expression, which becomes evident only after pathogen attack. Both SAR and ISR are effective against a broad spectrum of virulent plant pathogens.

They showed that two different alleles of the Arabidopsis npr1 mutant (npr1-1 and npr1-3) behaved differently in terms of transcriptome changes upon infection by $P$. syringae ${ }^{53}$. The npr1-1 mutant, which has a mutation in a crucial ankyrin-repeat domain, was affected in the expression of SA- as well as JA/ET-dependent genes. However, the npr1-3 mutant, which produces a truncated cytoplasmatically localized NPR1 protein ${ }^{30}$, was only affected in SA-dependent gene expression, which suggests that the cytoplasmatic function of NPR1 plays a role in the control of JA/ET-dependent responses. In agreement with this, the antagonistic effect of SA on JA-responsive gene expression was much less affected in npr1-3 than in npr1-1 (ref. 75). This suggests a model in which the cytosolic function of NPR1 plays a role in SA-JA crosstalk, and in which the nuclear function of NPR1 plays a role in the activation of SA-responsive genes.

Recently, it was demonstrated that ET bypasses the need of NPR1 in SA-JA crosstalk, while it enhances NPR1-dependent, SA-responsive $P R-1$ expression ${ }^{75}$. These findings indicate that the final outcome of the SA-JA signal interaction during the complex interaction of plants with their attackers can be shaped by ET. Indeed, the antagonistic effect of SA on JA-dependent resistance against feeding by ET-noninducing 
Figure 5 Simplified schematic representation of the SA, JA and ET signaling pathways. (a) SA mediates a change in the cellular redox potential, resulting in the reduction of the NPR1 oligomer to its active monomeric form. Monomeric NPR1 is then translocated into the nucleus where it functions as a transcriptional co-activator of SA-responsive genes, such as $P R-1$, by enhancing the binding of TGA transcription factors to $S A$-responsive promoter elements 4,30 . (b) In the JA signaling cascade, the E3 ubiquitin ligase $\mathrm{SCF}^{\mathrm{COI} 1}$ complex and jasmonate ZIM-domain (JAZ) proteins form a complex that represses transcription of JA-responsive genes ${ }^{49}$. Upon accumulation of JA, JA-isoleucine (JA-Ile) binds to the F-box protein COI1 in the $\mathrm{SCF}^{\mathrm{CO} 1}$ complex, after which the JAZ proteins are ubiquitinated and subsequently degraded through the $26 \mathrm{~S}$ proteasome. This results in the activation of JA-responsive genes through the action of transcription factors such as MYC2, ERF1 and ORA59 (refs. 63,77). (c) In the ET signaling cascade, the gaseous hormone ET is perceived by plasma membrane receptors such as ETR 1 (ref. 48). Genetically, these receptors are negative regulators of the ET response, because in the absence of ET they maintain the negative regulatory role of CTR1, which represses the positive regulator EIN2. Upon perception of ET, the repression of ET signaling by CTR1 is relieved, allowing downstream signaling through EIN2. Subsequently, critical positive regulators of ET-responsive gene expression, such as EIN3, become active because the E3 ubiquitin ligase SCFEBF1/2-dependent 26S proteasome degradation of these proteins becomes inhibited. EIN3-like transcription factors activate transcription factors such as ERF1, resulting in the expression of downstream ET-responsive genes. thrips (Frankliniella occidentalis) was controlled by NPR1 (ref. 75). By contrast, SA-mediated suppression of JA-dependent resistance against the JA- and ET-inducing necrotroph A. brassicicola functioned partly independently of NPR1. This highlights the tight connections between the SA, JA and ET pathways in the defense signaling network and provides further evidence for a dual role for NPR1 in regulating SA-mediated activation of SA-dependent defenses on the one hand, and SA-mediated suppression of JA-dependent defenses on the other hand. NPR1 has been implicated in several other JA/ET-dependent defense responses, including beneficial rhizobacteria-mediated ISR (ref. 34) and JA/ET-dependent resistance against the soil-borne fungus Verticillium longisporum ${ }^{76}$. However, the molecular mechanisms by which NPR1 exerts its role in these JA/ET-dependent defenses remains to be elucidated.

JA-ET. In many cases, the interaction between JA and ET signaling is a synergistic one. A classic example is the regulation of the Arabidopsis plant defensin gene PDF1.2, which requires concomitant activation of the JA and ET response pathways ${ }^{51}$. ERFs are members of the large plant-specific APETALA2/ETHYLENE RESPONSE FACTOR (AP2/ ERF) superfamily of transcription factors. Two members of this superfamily, ERF1 and ORA59, emerged as principal integrators of the JA and ET signaling pathways ${ }^{77,78}$ (Fig. 6). The expression of both ERF1 and ORA59 is induced by JA and ET and can be activated synergistically by both hormones. In addition, overexpression of the transcription factor genes ERF1 or ORA59 in the JA-insensitive mutant coil, or ERF1 in the ET-insensitive mutant ein2, constitutively activated the PDF1.2 gene, which indicates that these transcription factors are important nodes of convergence of JA and ET signaling.

Like ERF1 and ORA59, the basic helix-loop-helix leucine zipper transcription factor MYC2 (originally called JIN1, for JASMONATE INSENSITIVE1) has been demonstrated to play an important role in the regulation of JA-responsive genes ${ }^{63}$ (Fig. 6). Upon induction of the JA pathway, MYC2 differentially regulates two distinct classes of JA-responsive genes. MYC2 functions as a positive regulator of JA-responsive genes such as VSP2 and LOX2, whereas it acts as a negative regulator of JA/ET-responsive genes such as PDF1.2 that are activated by ERFs ${ }^{79}$. Hence, when the JA response is activated

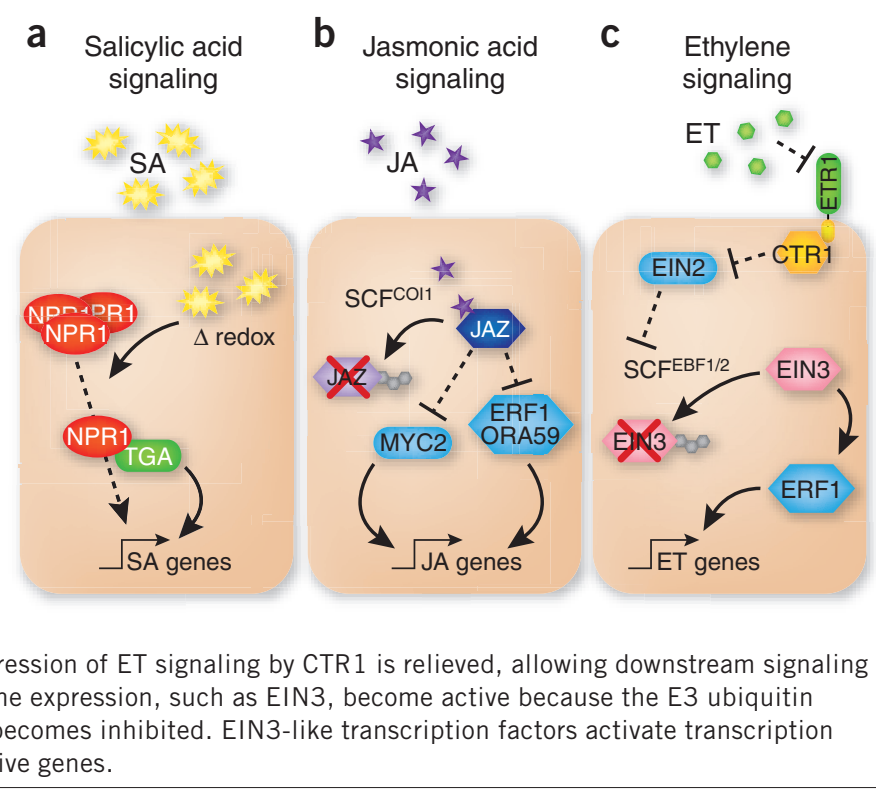

in combination with ET, the ERF branch of the JA response is activated, while the MYC2 branch of the JA response is activated when ET is absent. ABA was shown to play a role in favoring the MYC2dependent branch of the JA response ${ }^{80}$. Such a differential JA response was clearly apparent when the whole-genome expression profile of Arabidopsis was analyzed after infection with the necrotroph A. brassicicola, which induces the production of both JA and ET, or after infestation with herbivorous Western flower thrips (F. occidentalis), which stimulates the biosynthesis of JAs but not that of $\mathrm{ET}^{17}$. Hence, the interplay between the ERFs and MYC2 may allow the plant to activate the set of JA-responsive genes that is required for optimal defense against the attacker encountered. Though JA signaling mutants are generally more susceptible to necrotropic pathogens such as Botrytis cinerea $^{18,26}$, the Arabidopsis mutant jin1/myc2 showed enhanced resistance against this pathogen ${ }^{81}$. Given that the inhibitory effect of MYC2 on the ERF branch of the JA response is relieved in the jin $1 /$ myc2 mutant, the enhanced resistance against $B$. cinerea may be caused by a potentiated expression of ERF-dependent defenses in this mutant. Interestingly, MYC2 is also implicated in systemic immunity triggered by SAR-inducing bacteria ${ }^{82}$ and in the regulation of genes that show a primed expression pattern after pathogen infection in plants expressing JA/ET-dependent rhizobacteria-mediated ISR (ref. 38). Moreover, MYC2 is involved in mediating the suppression of SA-dependent defenses by coronatine, a phytotoxic virulence factor of $P$. syringae that mimics the action of $\mathrm{JAs}^{81,83}$ (see below), which makes this transcription factor an important node in the SA-JA-ET signaling network.

ET-SA. ET has been demonstrated to be an important modulator of the plant's defense response to pathogen and insect attack ${ }^{3,6}$. For instance, from a study with ET-insensitive (Tetr) tobacco plants, it was concluded that ET is essential for the onset of SA-dependent SAR that is triggered upon infection by tobacco mosaic virus ${ }^{84}$. Moreover, ET was shown to enhance the response of Arabidopsis to SA, resulting in a potentiated expression of the SA-responsive marker gene $P R-1$ (refs. 85,86). This synergistic effect of ET on SA-induced PR-1 expression was blocked in the ET-insensitive mutant ein2 (ref. 86), which indicates that the modulation of the SA pathway by ET is EIN2 
dependent and thus functions through the ET signaling pathway (Fig. 6). Further evidence for SA-ET crosstalk came from the network topology study of Glazebrook et al. in which the global expression profiles of $P$. syringae-infected Arabidopsis wild-type and signaling-defective mutant plants were analyzed $^{53}$. This study showed extensive crosstalk between the SA and ET signaling pathways, as evidenced by the fact that the expression of many SA-responsive genes was significantly affected in the ein 2 mutant background.

\section{Hormone pathways connected to the SA- JA-ET backbone}

Though the SA, JA and ET response pathways serve as the backbone of the induced defense signaling network, studies in Arabidopsis demonstrated that other hormone response pathways feed into it (Fig. 6). ABA is commonly associated with plant development and abiotic stress, but its role in biotic stress is becoming increasingly evident ${ }^{7,8}$. ABA is connected to the SA-JA-ET network, as it was shown to attenuate JA/ET-dependent gene expression ${ }^{80}$ and to affect JA biosynthesis and resistance against JA-inducing necrotrophic pathogens ${ }^{87,88}$. Moreover, ABA was demonstrated to antagonize the onset of SA-dependent defenses and $\mathrm{SAR}^{89,90}$. Interestingly, $\mathrm{NaCl}$-activated abiotic stress had a similar suppressive effect on the SA-dependent SAR in Arabidopsis ${ }^{89}$. Conversely, activation of SAR suppressed the expression of ABA-related genes, which indicates that $\mathrm{ABA}$ serves as an important regulator that functions at the crossroad of abiotic and biotic stress responses.

Auxins play a role in virtually every stage of plant development. The auxin response pathway is connected to the SA-JA-ET signaling network in different ways. For instance, auxin has been demonstrated to affect JA biosynthesis ${ }^{91}$ and the expression of genes involved in JA production $^{92}$. Second, auxin signaling was shown to promote disease susceptibility to P. syringae ${ }^{9,93}$, a process that can be counteracted by $\mathrm{SA}^{10}$. Whole-genome expression profiling revealed that SA interferes with auxin responses by global repression of auxin-related genes, including the auxin receptor gene TIR1. The inhibitory effect of SA on auxin responses stimulated effective defenses against the (hemi) biotrophic pathogens $H$. arabidopsidis and $P$. syringae, resulting in heightened resistance to these pathogens ${ }^{10}$. Hence, the antagonistic effect of SA on auxin signaling seems to be an intrinsic part of SA-dependent resistance against (hemi)biotrophs.

Recently, gibberellins were shown to hook up to the SA-JA-ET network as well. Gibberellins are hormones that control plant growth by regulating the degradation of growth-repressing DELLA proteins. Navarro et al. demonstrated that DELLA proteins promote susceptibility to biotrophic pathogens and resistance to necrotrophic pathogens by modulating the relative strength of the SA and JA signaling pathways ${ }^{11}$. Hence, it was postulated that by regulating the stability of DELLA proteins, gibberellins are able to modulate the SA-JA-ET network and affect the final outcome of the immune response.
Cytokinins often work in concert with auxins in processes such as cell division and differentiation of plant tissues. They are linked to the response of plants to biotrophic pathogens that alter the host's physiology ${ }^{12}$, such as Plasmodiophora brassicae, which causes aberrant root growth (club roots) in Brassica species ${ }^{13}$. However, little is known about their connection with the SA-JA-ET network.

Brassinosteroids play a key role in cell expansion and division, differentiation and reproductive development. When applied exogenously, they are able to induce a broad-spectrum disease resistance ${ }^{14}$. Brassinosteroids are perceived by the receptor BRI1, which interacts with the receptor-like kinase BAK1 to initiate an intracellular signaling cascade that regulates growth- and development-related processes ${ }^{94}$. Interestingly, BAK1 also interacts with receptors that recognize PAMPs, such as bacterial flagellin, resulting in the initiation of innate immunity ${ }^{95,96}$. Pathogen-specific effectors of $P$. syringae have been shown to interfere with this process by binding to BAK1 themselves; they consequently impede the host immune response ${ }^{15}$. However, the role of BAK1 in the innate immune response seems to be independent of the function of BAK1 in brassinosteroid signaling ${ }^{97}$. Hence, a connection between brassinosteroid signaling and the SA-JA-ET network remains to be established.

\section{Decoy strategies of the attacker}

Interplay between hormonal signaling pathways may provide the plant with a powerful regulatory potential; it is also a possible target 
for plant attackers to manipulate the plant defense signaling network for their own benefit ${ }^{45,65}$. A classic example of a pathogen that ingeniously makes use of the plant's hormonal signaling network is the Crown Gall disease-causing pathogen Agrobacterium tumefaciens, which is often used for the production of genetically modified transgenic plants ${ }^{98}$. Agrobacterium genetically transforms its host plant by transferring a piece of the tumor-inducing ( $\mathrm{Ti}$ ) plasmid into the host's genome. The transferred T-DNA carries genes that encode for the production of auxins and cytokinins, resulting in uncontrolled host cell proliferation from which the pathogen can benefit. The fact that pathogens can produce phytohormone-like compounds as part of their colonization strategy has been well documented ${ }^{8,65}$. For instance, the necrotrophic pathogen $B$. cinerea is known to produce ET (ref. 99), whereas numerous bacteria have been shown to produce auxin, possibly to promote virulence ${ }^{100}$. An interesting example is the source of the name of the phytohormone gibberellin, the necrotrophic fungus Gibberella fujikuroi ${ }^{65}$. This fungus was demonstrated to produce significant amounts of gibberellin, possibly to disable JA-dependent necrotroph resistance through gibberellinmediated destabilization of DELLA proteins that play a role in controlling the balance between SA- and JA-dependent defenses ${ }^{11}$.

A well-studied example of a pathogen that suppresses the host's innate immune response by manipulating phytohormone homeostasis is the bacterial pathogen P. syringae ${ }^{101}$. P. syringae is able to inject different virulence effector proteins into the host cell via the type III protein secretion system. Several of these virulence factors have been demonstrated to suppress host defense responses (Fig. 6). For instance, the type III effector AvrRpt 2 was shown to promote bacterial virulence in a compatible Arabidopsis host by altering the host's auxin physiology, resulting in enhanced disease ${ }^{93}$. In addition, type III effectors of $P$. syringae were shown to activate the ABA signaling pathway as a major virulence strategy, resulting in the suppression of host immunity and enhanced disease ${ }^{102}$. As a counter measure, plants activate SA-dependent defenses that are effective against this pathogen and are able to neutralize some of the immune-suppressive effects, such as the effector-induced auxin response ${ }^{10}$. However, the pathogen is in turn able to suppress SA-dependent defenses. It does this, for instance, through the action of the virulence factor HopI1, which is able to suppress SA accumulation in the chloroplast ${ }^{103}$. In addition, $P$. syringae produces the phytotoxin coronatine, which functions as a JA mimic and suppresses SA-dependent defenses, thereby promoting susceptibility of the plant to this pathogen ${ }^{104,105}$. Interestingly, the degree of interplay between the SA- and JA-dependent defenses differs among Arabidopsis accessions ${ }^{54,106}$, which suggests intraspecific variability in how these defense pathways cross-communicate. In an ecological context, this natural variation in pathway crosstalk might be instrumental in avoiding the decoy strategies that are used by the plant's enemies.

\section{Concluding remarks}

To elucidate molecular mechanisms involved in plant immunity, Arabidopsis has been demonstrated to be an excellent model species. In many Arabidopsis-pathogen interactions, the roles of small-molecule hormones in the regulation of plant immunity have been demonstrated, and the underlying mechanisms have been uncovered. Nonetheless, nature is far more complex. For instance, although the role of SA in biotroph resistance and the role of JAs in necrotroph resistance may be clear in many plant-pathogen interactions, there are plenty of exceptions to this rule ${ }^{18}$. The same holds true for the significance of pathway crosstalk, pathogen-produced hormones and pathogen-mediated manipulation of hormone signaling pathways for the final outcome of the host immune response. As plant defense mechanisms have evolved during the co-evolutionary arms race between plants and their attackers and have come with costs in addition to benefits, insights into their significance for plant survival should ideally come from ecological studies. Hence, a future challenge for research on plant immunity is to put laboratory findings to the test in an ecological context ${ }^{45}$.

On the other hand, there is a clear need to understand how complex high-dimensional signal interactions are translated into a definite coordinated defense response that is effective against the type of pathogen that the plant is encountering. The fact that plant growth regulators are so heavily involved in the orchestration of the plant immune response suggests that developmental and defense signaling networks are interconnected. The enormous amounts of data that plant biologists from different fields produced in the past years through the availability of large-scale genomics datasets and genome-wide mutagenesis libraries calls upon a systems approach in which computational biology and bioinformatics techniques enable researchers to integrate and comprehend these complex datasets ${ }^{107,108}$. Research on networking by smallmolecule hormones in plant immunity has reached the phase in which systems biology will move the field beyond the reductionist approach toward the identification of emergent properties that otherwise would remain undiscovered. Because the kinetics of phytohormone biosynthesis and the signature of the blend of alarm signals produced upon pathogen attack are pivotal for the final outcome of the plant-pathogen interaction, the field of chemical biology will be highly instrumental in uncovering how small-molecule hormones regulate the plant's immune signaling circuitry.

\section{ACKNOWLEDGMENTS}

The authors of this review are supported by VICI grant no. 865.04 .002 of the Earth and Life Sciences Foundation, which is subsidized by the Netherlands Organization of Scientific Research (NWO); the Centre for BioSystems Genomics (CBSG), which is part of the Netherlands Genomics Initiative of the NWO; and project T3-103 of the Top Institute Pharma.

Published online at http://www.nature.com/naturechemicalbiology/ Reprints and permissions information is available online at http://npg.nature.com/ reprintsandpermissions/

1. Buchanan, B.B., Gruissem, W. \& Jones, R.L. Biochemistry \& Molecular Biology of Plants 1367 (American Society of Plant Physiologists, Rockville, Maryland, USA, 2000).

2. Pozo, M.J., Van Loon, L.C. \& Pieterse, C.M.J. Jasmonates-signals in plant-microbe interactions. J. Plant Growth Regul. 23, 211-222 (2004).

3. Van Loon, L.C., Geraats, B.P.J. \& Linthorst, H.J.M. Ethylene as a modulator of disease resistance in plants. Trends Plant Sci. 11, 184-191 (2006).

4. Loake, G. \& Grant, M. Salicylic acid in plant defence - the players and protagonists Curr. Opin. Plant Biol. 10, 466-472 (2007).

5. Howe, G.A. Jasmonates as signals in the wound response. J. Plant Growth Regul. 23 223-237 (2004).

6. Von Dahl, C.C. \& Baldwin, I.T. Deciphering the role of ethylene in plant-herbivore interactions. J. Plant Growth Regul. 26, 201-209 (2007).

7. Asselbergh, B., De Vleesschauwer, D. \& Höfte, M. Global switches and fine-tuningABA modulates plant pathogen defense. Mol. Plant Microbe Interact. 21, 709-719 (2008).

8. Mauch-Mani, B. \& Mauch, F. The role of abscisic acid in plant-pathogen interactions. Curr. Opin. Plant Biol. 8, 409-414 (2005).

9. Navarro, L. et al. A plant miRNA contributes to antibacterial resistance by repressing auxin signaling. Science 312, 436-439 (2006)

10. Wang, D., Pajerowska-Mukhtar, K., Hendrickson Culler, A. \& Dong, X. Salicylic acid inhibits pathogen growth in plants through repression of the auxin signaling pathway. Curr. Biol. 17, 1784-1790 (2007).

11. Navarro, L. et al. DELLAs control plant immune responses by modulating the balance of jasmonic acid and salicylic acid signaling. Curr. Biol. 18, 650-655 (2008).

12. Walters, D.R. \& McRoberts, N. Plants and biotrophs: a pivotal role for cytokinins? Trends Plant Sci. 11, 581-586 (2006).

13. Siemens, J. et al. Transcriptome analysis of Arabidopsis clubroots indicate a key role for cytokinins in disease development. Mol. Plant Microbe Interact. 19, 480-494 (2006).

14. Nakashita, $\mathrm{H}$. et al. Brassinosteroid functions in a broad range of disease resistance in tobacco and rice. Plant J. 33, 887-898 (2003). 
15. Shan, L.B. et al. Bacterial effectors target the common signaling partner BAK1 to disrupt multiple MAMP receptor-signaling complexes and impede plant immunity. Cell Host Microbe 4, 17-27 (2008).

16. Walters, D. \& Heil, M. Costs and trade-offs associated with induced resistance. Physiol. Mol. Plant Pathol. 71, 3-17 (2007).

17. De Vos, M. et al. Signal signature and transcriptome changes of Arabidopsis during pathogen and insect attack. Mol. Plant Microbe Interact. 18, 923-937 (2005).

18. Glazebrook, J. Contrasting mechanisms of defense against biotrophic and necrotrophic pathogens. Annu. Rev. Phytopathol. 43, 205-227 (2005)

19. Jones, J.D.G. \& Dangl, J.L. The plant immune system. Nature 444, 323-329 (2006).

20. Göhre, V. \& Robatzek, S. Breaking the barriers: microbial effector molecules subvert plant immunity. Annu. Rev. Phytopathol. 46, 189-215 (2008),

21. Nürnberger, T. \& Kemmerling, B. Pathogen-associated molecular patterns (PAMP) and PAMP-triggered immunity. Annu. Plant Rev. 34, 16-47 (2009).

22. Chisholm, S.T., Coaker, G., Day, B. \& Staskawicz, B.J. Host-microbe interactions: shaping the evolution of the plant immune response. Cell 124, 803-814 (2006).

23. Schwessinger, B. \& Zipfel, C. News from the frontline: recent insights into PAMPtriggered immunity in plants. Curr. Opin. Plant Biol. 11, 389-395 (2008).

24. Tsuda, K., Sato, M., Glazebrook, J., Cohen, J.D. \& Katagiri, F. Interplay between MAMPtriggered and SA-mediated defense responses. Plant J. 53, 763-775 (2008).

25. De Wit, P.J.G.M. Pathogen avirulence and plant resistance: a key role for recognition. Trends Plant Sci. 2, 452-458 (1997).

26. Thomma, B.P.H.J., Penninckx, I.A.M.A., Broekaert, W.F. \& Cammue, B.P.A. The complexity of disease signaling in Arabidopsis. Curr. Opin. Immunol. 13, 63-68 (2001).

27. Durrant, W.E. \& Dong, X. Systemic acquired resistance. Annu. Rev. Phytopathol. 42 , 185-209 (2004).

28. Van Loon, L.C., Rep, M. \& Pieterse, C.M.J. Significance of inducible defense-related proteins in infected plants. Annu. Rev. Phytopathol. 44, 135-162 (2006).

29. Mishina, T.E. \& Zeier, J. Pathogen-associated molecular pattern recognition rather than development of tissue necrosis contributes to bacterial induction of systemic acquired resistance in Arabidopsis. Plant J. 50, 500-513 (2007).

30. Dong, X. NPR1, all things considered. Curr. Opin. Plant Biol. 7, 547-552 (2004).

31. Vlot, A.C., Klessig, D.F. \& Park, S.-W. Systemic acquired resistance: the elusive signal(s). Curr. Opin. Plant Biol. 11, 436-442 (2008).

32. Van Loon, L.C., Bakker, P.A.H.M. \& Pieterse, C.M.J. Systemic resistance induced by rhizosphere bacteria. Annu. Rev. Phytopathol. 36, 453-483 (1998).

33. Pozo, M.J. \& Azcon-Aguilar, C. Unraveling mycorrhiza-induced resistance. Curr. Opin. Plant Biol. 10, 393-398 (2007).

34. Van Wees, S.C.M., Van der Ent, S. \& Pieterse, C.M.J. Plant immune responses triggered by beneficial microbes. Curr. Opin. Plant Biol. 11, 443-448 (2008).

35. Bakker, P.A.H.M., Pieterse, C.M.J. \& Van Loon, L.C. Induced systemic resistance by fluorescent Pseudomonas spp. Phytopathology 97, 239-243 (2007).

36. Van der Ent, S. et al. MYB72 is required in early signaling steps of rhizobacteria-induced systemic resistance in Arabidopsis. Plant Physiol. 146, 1293-1304 (2008).

37. Conrath, U. et al. Priming: getting ready for battle. Mol. Plant Microbe Interact. 19, 1062-1071 (2006).

38. Pozo, M.J., Van der Ent, S., Van Loon, L.C. \& Pieterse, C.M.J. Transcription factor MYC2 is involved in priming for enhanced defense during rhizobacteria-induced systemic resistance in Arabidopsis thaliana. New Phytol. 180, 511-523 (2008).

39. Ton, J., Van Pelt, J.A., Van Loon, L.C. \& Pieterse, C.M.J. Differential effectiveness of salicylate-dependent and jasmonate/ethylene-dependent induced resistance in Arabidopsis. Mol. Plant Microbe Interact. 15, 27-34 (2002).

40. Van Oosten, V.R. et al. Differential effectiveness of microbially induced resistance against herbivorous insects in Arabidopsis. Mol. Plant Microbe Interact. 21, 919-930 (2008).

41. Stout, M.J., Thaler, J.S. \& Thomma, B.P.H.J. Plant-mediated interactions between pathogenic microorganisms and herbivorous arthropods. Annu. Rev. Entomol. 51, 663-689 (2006).

42. Poelman, E.H., van Loon, J.J.A. \& Dicke, M. Consequences of variation in plant defense for biodiversity at higher trophic levels. Trends Plant Sci. 13, 534-541 (2008).

43. Van der Putten, W.H., Vet, L.E.M., Harvey, J.A. \& Wäckers, F.L. Linking above- and belowground multitrophic interactions of plants, herbivores, pathogens, and their antagonists. Trends Ecol. Evol. 16, 547-554 (2001).

44. Reymond, P. \& Farmer, E.E. Jasmonate and salicylate as global signals for defense gene expression. Curr. Opin. Plant Biol. 1, 404-411 (1998).

45. Pieterse, C.M.J. \& Dicke, M. Plant interactions with microbes and insects: from molecular mechanisms to ecology. Trends Plant Sci. 12, 564-569 (2007).

46. Kunkel, B.N. \& Brooks, D.M. Cross talk between signaling pathways in pathogen defense. Curr. Opin. Plant Biol. 5, 325-331 (2002).

47. Bostock, R.M. Signal crosstalk and induced resistance: straddling the line between cost and benefit. Annu. Rev. Phytopathol. 43, 545-580 (2005).

48. Kendrick, M.D. \& Chang, C. Ethylene signaling: new levels of complexity and regulation. Curr. Opin. Plant Biol. 11, 479-485 (2008).

49. Katsir, L., Chung, H.S., Koo, A.J.K. \& Howe, G.A. Jasmonate signaling: a conserved mechanism of hormone sensing. Curr. Opin. Plant Biol. 11, 428-435 (2008).

50. Doherty, H.M., Selvendran, R.R. \& Bowles, D.J. The wound response of tomato plants can be inhibited by aspirin and related hydroxy-benzoic acids. Physiol. Mol. Plant Pathol. 33, 377-384 (1988).

51. Penninckx, I.A.M.A., Thomma, B.P.H.J., Buchala, A., Métraux, J.-P. \& Broekaert, W.F. Concomitant activation of jasmonate and ethylene response pathways is required for induction of a plant defensin gene in Arabidopsis. Plant Cell 10, 2103-2113 (1998).

52. Katagiri, F. A global view of defense gene expression regulation-a highly interconnected signaling network. Curr. Opin. Plant Biol. 7, 506-511 (2004).

53. Glazebrook, J. et al. Topology of the network integrating salicylate and jasmonate signal transduction derived from global expression phenotyping. Plant J. 34, 217-228 (2003).

54. Koornneef, A. et al. Kinetics of salicylate-mediated suppression of jasmonate signaling reveal a role for redox modulation. Plant Physiol. 147, 1358-1368 (2008).

55. Spoel, S.H., Johnson, J.S. \& Dong, X. Regulation of tradeoffs between plant defenses against pathogens with different lifestyles. Proc. Natl. Acad. Sci. USA 104, 1884218847 (2007).

56. Spoel, S.H. et al. NPR1 modulates cross-talk between salicylate- and jasmonatedependent defense pathways through a novel function in the cytosol. Plant Cel/ 15, 760-770 (2003).

57. Schenk, P.M. et al. Coordinated plant defense responses in Arabidopsis revealed by microarray analysis. Proc. Natl. Acad. Sci. USA 97, 11655-11660 (2000).

58. Mur, L.A.J., Kenton, P., Atzorn, R., Miersch, O. \& Wasternack, C. The outcomes of concentration-specific interactions between salicylate and jasmonate signaling include synergy, antagonism, and oxidative stress leading to cell death. Plant Physiol. 140, 249-262 (2006).

59. Van Wees, S.C.M., De Swart, E.A.M., Van Pelt, J.A., Van Loon, L.C. \& Pieterse, C.M.J. Enhancement of induced disease resistance by simultaneous activation of salicylate- and jasmonate-dependent defense pathways in Arabidopsis thaliana. Proc. Natl. Acad. Sci. USA 97, 8711-8716 (2000).

60. Koornneef, A. \& Pieterse, C.M.J. Cross-talk in defense signaling. Plant Physiol. 146 839-844 (2008).

61. Spoel, S.H. \& Dong, X. Making sense of hormone crosstalk during plant immune responses. Cell Host Microbe 3, 348-351 (2008).

62. Kazan, K. \& Manners, J.M. Jasmonate signaling: toward an integrated view. Plant Physiol. 146, 1459-1468 (2008).

63. Lorenzo, O. \& Solano, R. Molecular players regulating the jasmonate signalling network. Curr. Opin. Plant Biol. 8, 532-540 (2005).

64. López, M.A., Bannenberg, G. \& Castresana, C. Controlling hormone signaling is a plant and pathogen challenge for growth and survival. Curr. Opin. Plant Biol. 11, 420-427 (2008).

65. Robert-Seilaniantz, A., Navarro, L., Bari, R. \& Jones, J.D.G. Pathological hormone imbalances. Curr. Opin. Plant Biol. 10, 372-379 (2007).

66. Petersen, M. et al. Arabidopsis map kinase 4 negatively regulates systemic acquired resistance. Cell 103, 1111-1120 (2000).

67. Brodersen, P. et al. Arabidopsis MAP kinase 4 regulates salicylic acid- and jasmonic acid/ethylene-dependent responses via EDS1 and PAD4. Plant J. 47, 532-546 (2006).

68. Kachroo, P., Shanklin, J., Shah, J., Whittle, E.J. \& Klessig, D.F. A fatty acid desaturase modulates the activation of defense signaling pathways in plants. Proc. Nat/. Acad. Sci. USA 98, 9448-9453 (2001).

69. Ndamukong, I. et al. SA-inducible Arabidopsis glutaredoxin interacts with TGA factors and suppresses JA-responsive PDF1.2 transcription. Plant J. 50, 128-139 (2007).

70. Li, J., Brader, G. \& Palva, E.T. The WRKY70 transcription factor: a node of convergence for jasmonate-mediated and salicylate-mediated signals in plant defense. Plant Cell 16, 319-331 (2004).

71. Kachroo, P., Kachroo, A., Lapchyk, L., Hildebrand, D. \& Klessig, D.F. Restoration of defective cross talk in ssi2 mutants: Role of salicylic acid, jasmonic acid, and fatty acids in SSI2-mediated signaling. Mol. Plant Microbe Interact. 16, 1022-1029 (2003).

72. Mou, Z., Fan, W.H. \& Dong, X.N. Inducers of plant systemic acquired resistance regulate NPR 1 function through redox changes. Cell 113, 935-944 (2003).

73. Pieterse, C.M.J. \& Van Loon, L.C. NPR1: the spider in the web of induced resistance signaling pathways. Curr. Opin. Plant Biol. 7, 456-464 (2004).

74. Yuan, Y. et al. Functional analysis of rice NPR1-like genes reveals that OsNPR1/ $\mathrm{NH} 1$ is the rice orthologue conferring disease resistance with enhanced herbivore susceptibility. Plant Biotechnol. J. 5, 313-324 (2007).

75. Leon-Reyes, A. et al. Ethylene modulates the role of NONEXPRESSOR OF PATHOGENESIS-RELATED GENES 1 in cross talk between salicylate and jasmonate signaling. Plant Physiol. 149, 1797-1809 (2009).

76. Johansson, A., Staal, J. \& Dixelius, C. Early responses in the Arabidopsis-Verticillium longisporum pathosystem are dependent on NDR1, JA- and ET-associated signals via cytosolic NPR1 and RFO1. Mol. Plant Microbe Interact. 19, 958-969 (2006).

77. Pré, M. et al. The AP2/ERF domain transcription factor ORA59 integrates jasmonic acid and ethylene signals in plant defense. Plant Physiol. 147, 1347-1357 (2008).

78. Lorenzo, O., Piqueras, R., Sánchez-Serrano, J.J. \& Solano, R. ETHYLENE RESPONSE FACTOR 1 integrates signals from ethylene and jasmonate pathways in plant defense. Plant Cell 15, 165-178 (2003).

79. Lorenzo, O., Chico, J.M., Sanchez-Serrano, J.J. \& Solano, R. JASMONATEINSENSITIVE1 encodes a MYC transcription factor essential to discriminate between different jasmonate-regulated defense responses in Arabidopsis. Plant Cell 16, 1938-1950 (2004)

80. Anderson, J.P. et al. Antagonistic interaction between abscisic acid and jasmonateethylene signaling pathways modulates defense gene expression and disease resistance in Arabidopsis. Plant Cell 16, 3460-3479 (2004).

81. Nickstadt, A. et al. The jasmonate-insensitive mutant jin 1 shows increased resis- 
tance to biotrophic as well as necrotrophic pathogens. Mol. Plant Pathol. 5, 425-434 (2004).

82. Truman, W., Bennett, M.H., Kubigsteltig, I., Turnbull, C. \& Grant, M. Arabidopsis systemic immunity uses conserved defense signaling pathways and is mediated by jasmonates. Proc. NatI. Acad. Sci. USA 104, 1075-1080 (2007).

83. Laurie-Berry, N., Joardar, V., Street, I.H. \& Kunkel, B.N. The Arabidopsis thaliana JASMONATE INSENSITIVE 1 gene is required for suppression of salicylic aciddependent defenses during infection by Pseudomonas syringae. Mol. Plant Microbe Interact. 19, 789-800 (2006).

84. Verberne, M.C., Hoekstra, J., Bol, J.F. \& Linthorst, H.J.M. Signaling of systemic acquired resistance in tobacco depends on ethylene perception. Plant J. 35, 27-32 (2003).

85. Lawton, K.A., Potter, S.L., Uknes, S. \& Ryals, J. Acquired resistance signal transduction in Arabidopsis is ethylene independent. Plant Cell 6, 581-588 (1994).

86. De Vos, M. et al. Herbivore-induced resistance against microbial pathogens in Arabidopsis. Plant Physiol. 142, 352-363 (2006).

87. Adie, B.A.T. et al. ABA is an essential signal for plant resistance to pathogens affecting JA biosynthesis and the activation of defenses in Arabidopsis. Plant Cell 19, 1665-1681 (2007).

88. Flors, V. et al. Interplay between JA, SA and ABA signalling during basal and induced resistance against Pseudomonas syringae and Alternaria brassicicola. Plant J. 54, 81-92 (2008).

89. Yasuda, M. et al. Antagonistic interaction between systemic acquired resistance and the abscisic acid-mediated abiotic stress response in Arabidopsis. Plant Cell 20, 1678-1692 (2008).

90. Mohr, P.G. \& Cahill, D.M. Suppression by ABA of salicylic acid and lignin accumulation and the expression of multiple genes, in Arabidopsis infected with Pseudomonas syringae pv. tomato. Funct. Integr. Genomics 7, 181-191 (2007).

91. Nagpal, P. et al. Auxin response factors ARF6 and ARF8 promote jasmonic acid production and flower maturation. Development 132, 4107-4118 (2005).

92. Liu, J. \& Wang, X.-J. An integrative analysis of the effects of auxin on jasmonic acid biosynthesis in Arabidopsis thaliana. J. Integr. Plant Biol. 48, 99-103 (2006).

93. Chen, Z.Y. et al. Pseudomonas syringae type III effector AvrRpt2 alters Arabidopsis thaliana auxin physiology. Proc. Natl. Acad. Sci. USA 104, 20131-20136 (2007).
94. Belkhadir, Y. \& Chory, J. Brassinosteroid signaling: a paradigm for steroid hormone signaling from the cell surface. Science 314, 1410-1411 (2006).

95. Chinchilla, D. et al. A flagellin-induced complex of the receptor FLS2 and BAK1 initiates plant defence. Nature 448, 497-500 (2007).

96. Heese, A. et al. The receptor-like kinase SERK3/BAK1 is a central regulator of innate immunity in plants. Proc. Natl. Acad. Sci. USA 104, 12217-12222 (2007).

97. Kemmerling, B. et al. The BRI1-associated kinase 1, BAK1, has a brassinolideindependent role in plant cell-death control. Curr. Biol. 17, 1116-1122 (2007).

98. Tzfira, T. \& Citovsky, V. Agrobacterium-mediated genetic transformation of plants: biology and biotechnology. Curr. Opin. Biotechnol. 17, 147-154 (2006).

99. Cristescu, S.M., De Martinis, D., Hekkert, S.T., Parker, D.H. \& Harren, F.J.M Ethylene production by Botrytis cinerea in vitro and in tomatoes. Appl. Environ. Microbiol. 68, 5342-5350 (2002).

100. Spaepen, S., Vanderleyden, J. \& Remans, R. Indole-3-acetic acid in microbial and microorganism-plant signaling. FEMS Microbiol. Rev. 31, 425-448 (2007).

101. Nomura, K., Melotto, M. \& He, S.-Y. Suppression of host defense in compatible plantPseudomonas syringae interactions. Curr. Opin. Plant Biol. 8, 361-368 (2005).

102. de Torres-Zabala, M. et al. Pseudomonas syringae pv. tomato hijacks the Arabidopsis abscisic acid signalling pathway to cause disease. EMBO J. 26, 1434-1443 (2007).

103. Jelenska, J. et al. A J domain virulence effector of Pseudomonas syringae remodels host chloroplasts and suppresses defenses. Curr. Biol. 17, 499-508 (2007).

104. Brooks, D.M., Bender, C.L. \& Kunkel, B.N. The Pseudomonas syringae phytotoxin coronatine promotes virulence by overcoming salicylic acid-dependent defences in Arabidopsis thaliana. Mol. Plant Pathol. 6, 629-639 (2005).

105. Uppalapati, S.R. et al. The phytotoxin coronatine contributes to pathogen fitness and is required for suppression of salicylic acid accumulation in tomato inoculated with Pseudomonas syringae pv. tomato DC3000. Mol. Plant Microbe Interact. 20, 955-965 (2007).

106. Traw, M.B., Kim, J., Enright, S., Cipollini, D.F. \& Bergelson, J. Negative cross-talk between salicylate- and jasmonate-mediated pathways in the Wassilewskija ecotype of Arabidopsis thaliana. Mol. Ecol. 12, 1125-1135 (2003).

107. Scheres, B. \& Lipka, V. Plant cell biology—get your networks together. Curr. Opin Plant Biol. 10, 546-548 (2007).

108. Long, T.A., Rady, S.M. \& Benfey, P.N. Systems approaches to identifying gene regulatory networks in plants. Annu. Rev. Cell Dev. Biol. 24, 81-103 (2008). 